\title{
Multicenter Study of Lumen-apposing Metal Stents With or Without Pigtail in Endoscopic Ultrasound- guided Biliary Drainage for Malignant Obstruction- Bampi Trial: an Open-label, Randomized Controlled Trial Protocol.
}

\author{
Albert Garcia-Sumalla \\ Hospital Universitari de Bellvitge
}

\section{Carme Loras}

Hospital Universitari MutuaTerrassa

\section{Vicente Sanchiz}

Hospital Clínico Universitario de Valencia

\section{Rafael Pedraza-Sanz}

Hospital General Universitari de Castelló: Hospital General Universitari de Castello

\section{Enrique Vazquez-Sequeiros}

Hospital Ramon y Cajal: Hospital Universitario Ramon y Cajal

\section{Jose Ramon Aparicio}

Hospital General Universitario de Alicante: Hospital General Universitari d'Alacant

\section{Carlos De la Serna-Higuera}

Rio Hortega University Hospital: Hospital Universitario Rio Hortega

\section{Daniel Luna-Garcia}

Hospital Universitari de Bellvitge

\section{Xavier Andujar}

Hospital Universitari MútuaTerrassa: Hospital Universitari MutuaTerrassa

\section{Maria Capilla}

Hospital Clínico Universitario de Valencia

\section{Tatiana Barbera}

Hospital General Universitari de Castelló: Hospital General Universitari de Castello Jose Ramon Foruny-Olcina

Hospital Ramon y Cajal: Hospital Universitario Ramon y Cajal

\section{Belen Martinez-Moreno}

Hospital General Universitario de Alicante: Hospital General Universitari d'Alacant

\section{Miguel Dura}

Hospital Universitario Rio Hortega de Valladolid: Hospital Universitario Rio Hortega 


\section{Silvia Salord}

Hospital Universitari de Bellvitge

\section{Berta Laquente}

Hospital Duran i Reynals: Institut Catala d'Oncologia

\section{Cristian Tebe}

Bellvitge Institute for Biomedical Research: Institut d'Investigacio Biomedica de Bellvitge

\section{Sebastia Videla}

Bellvitge University Hospital: Hospital Universitari de Bellvitge

\section{Manuel Perez-Miranda}

Hospital Universitario Rio Hortega de Valladolid: Hospital Universitario Rio Hortega

Joan b Gornals ( $\nabla$ jgornals@bellvitgehospital.cat )

Hospital Universitari de Bellvitge https://orcid.org/0000-0001-8857-3556

\section{Research Article}

Keywords: Biliary drainage, Choledochoduodenostomy, Endoscopic ultrasound, Lumen-apposing metal stent, Malignant biliary obstruction, Plastic stent, Trial

Posted Date: February 4th, 2022

DOI: https://doi.org/10.21203/rs.3.rs-1286894/v1

License: (c) (i) This work is licensed under a Creative Commons Attribution 4.0 International License. Read Full License

Version of Record: A version of this preprint was published at Trials on February 25th, 2022. See the published version at https://doi.org/10.1186/s13063-022-06106-1. 


\section{Abstract}

Background: It is unclear whether the insertion of an axis-orienting double-pigtail plastic stent (DPS) through biliary lumen-apposing meal stent (LAMS) in EUS-guided choledochoduodenostomy (CDS) improves the stent patency. The aim of this study is to determine whether this technical variant offers a clinical benefit in EUS-guided biliary drainage (BD) for the management of distal malignant biliary obstruction.

Methods/design: This is a multicenter open-label, randomized controlled trial with two parallel groups. Eighty-four patients with malignant biliary obstruction will undergo EUS-BD (CDS type) using LAMS in 7 tertiary hospitals in Spain and will be randomized to the LAMS and LAMS plus DPS groups. The primary endpoint is the rate of recurrent biliary obstruction, as a stent dysfunction parameter, detected during follow-up. Secondary endpoints: technical and clinical success (reduction in bilirubin $>50 \%$ within 14 days of stent placement), safety, and others (number of reinterventions, time to biliary obstruction, prognostic factors, survival rate).

Discussion: The BAMPI trial has been designed to determine whether the addition of a coaxial axisorienting DPS through LAMS is superior to LAMS alone to prevent stent dysfunction.

Trial registration: ClinicalTrials.gov, NCT04595058. Registered on October 14, 2020.

\section{Background}

Transpapillary stenting by endoscopic retrograde cholangiopancreatography (ERCP) remains the gold standard treatment for malignant biliary obstruction (MBO), but may fail in up to $15 \%$ of cases, and it carries the risks of post-ERCP pancreatitis and stent dysfunction secondary to tumor ingrowth and/or overgrowth [1, 2]. For these reasons, since the emergence of Endoscopic Ultrasound-guided biliary drainage (EUS-BD) using electrocautery-enhanced (EE) lumen-apposing metal stents (LAMS), by creating a choledochoduodensotomy (CDS), it has been proposed as a viable alternative to ERCP [3, 4]. Firstly, as a rescue strategy after failed ERCP, in palliative scenarios, or even as a bridge to surgery $[5,6]$, and then recently, as a first-line modality for MBO in controlled trials [2, 7-9].

Although recent metanalyses and systematic reviews [10-14] have postulated that EUS-BD using LAMS has high technical and clinical success rates, some concerns exist regarding its safety, as non-negligible rates of adverse events $(\mathrm{AE})$ have been reported $[15,16]$. Recurrent biliary obstruction (RBO), as a stent dysfunction parameter, is a major issue to consider after EUS-BD using LAMS. Several cases of stent dysfunction (e.g., food or lithiasis impaction, sump syndrome, stent migration) have been documented due to a possible limitation in LAMS design, as the short length of the stent causes the distal flange to become impacted against the opposite biliary wall $[10,17,18]$. The insertion of a coaxial double-pigtail plastic stent (DPS) has been demonstrated to improve LAMS patency and bile flow, by maintaining a vertical orientation in the bile duct. But a recent retrospective study by our group did not encounter sufficient evidence to recommend its routine use [19]. 
For these reasons, a multicenter randomized controlled trial has been designed to assess whether a coaxial DPS within a biliary LAMS is superior to a single LAMS in EUS-BD (CDS type) for MBO.

\section{Methods/design}

The BAMPI (Biliary Apposing Metal Plgtail) trial is a multicenter, open-label, randomized controlled clinical trial with two parallel groups, and with a 1:1 allocation ratio. Eighty patients with MBO will be scheduled for EUS-BD in seven tertiary Spanish hospitals and will be randomized to the LAMS alone or the LAMS plus DPS group. We hypothesize the addition of a prophylactic DPS through LAMS to be associated with fewer episodes of RBO and, consequently, fewer biliary reinterventions (BRI).

Central ethical approval of the study protocol has been confirmed by the Comité Ético de Investigación Clínica (CEIC) del Hospital Universitari de Bellvitge-IDIBELL (ref approval no. ICPS024/20, on November, 2020) and we will not begin recruiting at other centers in the trial until local ethical approval has been obtained.

A checklist with the recommendations for Interventional Trials (SPIRIT) is attached as Additional file 1. SPIRIT-figure with schedule of enrolment, interventions, and assessments in the BAMPI trial (figure 1).

\section{Study population}

All patients admitted with distal MBO and clinical criteria that justify EUS-BD will be considered for consent. The investigator at each center will assess the inclusion of the patient in the study, and eligibility either as a first-line BD method, or as a rescue method after failed ERCP. The patient will be correctly informed by personnel knowledgeable about the specifics of the study, who will help to resolve any questions that may arise. The informed consent form will be signed prior to the procedure and a signed copy will be given to the patient. The patient has the right to opt out of the study at any time.

The inclusion and exclusion criteria are listed in Table 1. 
Inclusion and exclusion criteria.

Inclusion criteria:

Patients eligible for the trial must fulfil all the following at randomization

- Age 18 years or more

- Malignant biliary obstruction with clinical criteria that justifies EUS-guided biliary drainage.

- Capable of understanding and signing informed consent form.

- Understanding the type of study and complying with the follow-up of complementary tests during the study's duration.

\section{Exclusion criteria:}

Patients with any of the following will be excluded

- Pregnancy or breast-feeding.

- Severe coagulation disorder: INR > 1.5 not correctible with administration of plasma and/or platelets $<50,000 / \mathrm{mm} 3$.

- Maximum cross diameter of the CBD $<10 \mathrm{~mm}$.

- Another type of biliary drainage at the time of the procedure (cholecystostomy, percutaneous drainage, etc.).

- Failure to sign informed consent form.

- Intellectual handicap and unable to understand the nature and possible consequences of the study, unless there is a competent legal representative.

- Unable to adhere to subsequent follow-up requirements.

CBD common bile duct; EUS endoscopic ultrasound; INR international normalized ratio.

\section{Recruitment}

Principal investigators from each center will have the task of presenting strategies to promote enrolment and ensure the target sample size. Prior to the start of the study, a meeting with oncology and digestive surgery teams will be organized with the aim of creating collaboration bridges.

\section{Randomization and masking}

Patients will be enrolled in this trial by gastroenterologists, surgeons, oncologists, and endoscopists who will evaluate cases in the inpatient wards or outpatient consultation areas. Investigators of each center will be responsible for entering all necessary criteria to an online platform that will generate the randomization sequence, and participants will be randomized with an arbitrary number. 
A code list will be generated by our biostatistics department, using $R$ software ( $v$ 3.6.3) by randomization with a 1:1 randomization ratio, by blocks, stratified by age ( $<65$ years-old / $>65$ years-old) and by the presence of liver metastases. The assumption is made that liver metastasis may elevate bilirubin and cholestasis parameters without involving bile duct obstruction. Everyone will be assigned a randomization code along with the treatment that corresponds to it. Once the patient meets the eligibility criteria and has provided informed consent, we will proceed to the allocation of each participant centrally, ensuring allocation concealment, and based on the randomization list. To prevent different subject recruitment rates at the various hospitals from interfering in the development of the study, the entire population will be randomized in blocks of four between the two treatment possibilities.

\section{Procedural technique}

\section{Qualification of centers}

This clinical trial will be performed at the endoscopy unit of seven referral centers in Spain. Endoscopists will all be experienced in endoscopic intervention and therapeutics, such as stenting and EUS-guided transmural drainage. To avoid biases derived from the learning curve, only those centers with proven experience using biliary EE-LAMS (EUS-guided CDS) have been invited to participate. To objectify usage data, the database "NRPAL: National Registry of Lumen Apposing Metal Stents Incidences, NCT04059926", which collects data from all LAMS placed for one year, was checked. Minimum requirements were at least 12 LAMS placed in one year for any indication and a minimum experience of 7 EUS-BD using EE-LAMS. All the experienced endoscopists are members of the Spanish Society of Digestive Endoscopy (SEED).

\section{General description of the technique}

Treatment allocation is to EUS-BD with LAMS alone vs. LAMS plus DPS. All procedures will be performed by experienced endoscopists in EUS-guided transmural stenting. Procedures will be performed under deep sedation or tracheal intubation, in accordance with the directives of each center. Coagulation disorders will be corrected prior to the procedure. In case of INR $>1.5$ this will be corrected with the protocol of each institution. In all interventional procedures, $\mathrm{CO} 2$ will be used as an insufflation agent. Prophylactic antibiotic therapy will be given in accordance with institutional protocols.

\section{EUS-BD with EE-LAMS}

Each selected case will ensure a conclusive diagnosis of MBO. If necessary EUS-guided tissue acquisition (TA) will be performed previously to or immediately after the transmural drainage. The TA technique will be at the endoscopist's choice. Firstly, EUS oversight will be done with the purpose of ruling out features that could jeopardize a transmural BD (such as ascites, long distance common bile duct (CBD)transducer, or intervening vessels). The extrahepatic bile duct will be identified by using a linear echoendoscope (Fujifilm EG-580-UT, Olympus GF-UCT180), selecting an optimal point for carrying out the EUS-guided intervention. The long scope position will preferably be used to maintain stability and, if necessary, to ease advancing of a guidewire to intrahepatic direction. A small-medium sized LAMS $(6,8$, 
10-mm in diameter, and 8 or 10-mm in length, Hot-AXIOS ${ }^{\mathrm{Tm}}$ stent with an electrocautery-enhanced delivery system, Boston Scientific, Marlborough, MA, USA) will be used in all included cases.

The access step will be standardized in two different techniques, as explained in our previous report [19]: (i) freehand style (optional preloaded guidewire), with direct access to the bile duct, and (ii) classic technique, with initial puncture of the extrahepatic bile duct using a 19-gauge needle and advancing a standard guidewire, after which a LAMS is advanced over the guidewire.

In all cases, the biliary LAMS is inserted using the cautery system, and deployed under EUS-guidance without tract dilation. The use of fluoroscopy will be decided upon based on technical considerations and the endoscopist's opinion.

Selection of LAMS size will be based on bile duct diameter and availability.

\section{LAMS plus coaxial DPS procedure}

In those patients allocated in the LAMS-DPS cohort, a DPS (preferably 7Fr $\times 3,5$ or $7 \mathrm{~cm}$, Advanix, Boston Sc) will be placed coaxially through the LAMS, preferably with upward (intra-hepatic) orientation. This selection of small DPS sized should ease the advance and release by offering less friction.

\section{Additional interventions}

After the LAMS deployment, intra-stent dilation is permitted using balloons at the endoscopist's discretion.

Other endoscopic procedure such as EUS-guided TA, EUS-guided gastro-enterostomy, stenting (esophageal, duodenal), and EUS-guided celiac plexus blockage/neurolysis performed during the index procedure will be meticulously noted in a specific section in the electronic case report form (eCRF).

\section{Gallbladder option}

When a CDS is not possible or too risky, a BD rescue by EUS-guided gallbladder transmural drainage may be considered. It is important to note that this should only be offered in cases without an optimal endosonographic window for the creation of CDS (e.g., interposal vessels, CBD $<10 \mathrm{~mm}$, excessive $\mathrm{GI}$ tract-CBD distance, scope instability, others). It may be randomized in the same way (LAMS vs. LAMSDPS group) and followed up according to the protocol. This additional subgroup will be analyzed and documented, but outside of the trial's main outcomes.

\section{Additional comments}

No removal of the stents is contemplated due to the malignant condition of the participants. Noninpatients, they will remain in the center for a minimum of $24 \mathrm{~h}$ for clinical observation under clinical supervision.

In case of technical failure for any reason, alternative strategies will be decided upon with the aim of offering the best possible treatment to the patient. 


\section{Clinical evaluation and follow-up}

Data collection and calendar.

The collecting of clinical information of the patients will begin at the outset (baseline) and will continue with follow-up as established and defined in the study. Data will be collected at baseline visit, indexing procedure 24 hours later, and at days 14, 30, 90, 180, 270, and 1-year post-randomization. Collected data include primary, secondary, and additional endpoint data, demographics, comorbidities, oncological data, laboratory test findings, technical details, and clinical data during follow-up ((Figure 1 and 2).

AEs will be noted from the beginning of the test until the conclusion of follow-up by means of scheduled controls and will be handled and treated in accordance with the directives of the patient's medical team. All additional tests and interventions will be duly documented. Any instances of death during the followup will be investigated to rule out possible relation to the endoscopic procedure. Such occurrences will also be recorded in the CRF.

If there is clinical suspicion of obstruction or migration of the stent, an upper endoscopy will be carried out. Based on the findings of this procedure, the problem will be resolved in accordance with the directives of the intervening endoscopist. Any additional procedure or endoscopic intervention will be duly documented.

All data will be collected by research personnel knowledgeable in the use of the eCRF (data capturing software, redCAP). Collected patient data will be de-identified, meaning that personal information will not be stored in the main database. All data will be stored for 7 years after publication or as requested in standard operating procedure from individual sites. Only data relevant to the study as outlined in this protocol will be collected by the research team.

Due to the social and health crisis arising from the SARS-CoV-2 pandemic and taking into account the likely frailty of the candidates to be enrolled in the study, the protocol contemplates restrictions on physical presence visits. Only the indexing procedure and the 24 hours' post-procedure visits will require an on-site visit since a minimum one-day hospital admission is mandatory.

A BAMPI trial flowchart is provided (figure 2).

\section{Definitions}

Technical success is defined as successful placement of the stents (LAMS, DPS or both) between extrahepatic bile duct and gastrointestinal lumen by endoscopy, endosonography, and, optionally, fluoroscopy.

Clinical success is defined according to the TOKYO criteria as a $50 \%$ decrease in or normalization of total serum bilirubin level within 14 days of index procedure (stent placement). For cholangitis without obstructive jaundice (e.g., segmental cholangitis, cholecystitis), success can be defined as cessation of 
antibiotics or a $50 \%$ decrease in or normalization of levels of blood inflammatory markers within 14 days of stent insertion [20].

RBO is defined as a composite endpoint of stent dysfunction (either occlusion or migration). See Table 2 (outcomes) for complete information [20].

Table 2

Primary and secondary endpoints.

\section{Primary endpoint:}

- Rate of recurrent biliary obstruction (RBO) between the two strategies (LAMS with and without coaxial DPS)

*RBO is associated with a stent dysfunction (an endpoint of either occlusion ${ }^{\mathrm{a}}$ or migration ${ }^{\mathrm{b}}$ ). Tokyo criteria

- Clinical recurrence (jaundice, fever, suspicious colangitis, pruritus).

- Recurrence of cholestasis parameters (Any increase in GGT/ALP or bilirubin from its lowest level post-index procedure).

Both WITH evidence of biliary obstruction on imaging (dilation on US/CT/MRI) or endoscopic findings suggesting it $\mathrm{c}$.

Secondary endpoints:

- Technical success defined as successful stents (LAMS, DPS or either) between the extrahepatic biliary duct and the upper gastrointestinal tract determined by endoscopy, endosonography or fluoroscopy.

- Clinical success defined as $>50 \%$ decrease in bilirubin at 14 days from stent placement. For cholangitis, clinical success is defined as cessation of antibiotics or normalization of levels of blood inflammatory markers within 14 days of stent placement.

- Safety, as defined per the ASGE lexicon/Tokyo criteria for endoscopic AEs and divided into early adverse events (within 14 days of index procedure) and delayed $\mathrm{AE}$ (>14 days).

- Additional outcomes: t-RBO, BRI, procedure time and survival/mortality rates.

AE Adverse Event; ALP alkaline phosphatase; ASGE American Society of Gastrointestinal Endoscopy; BRI biliary reintervention; CT computed tomography; ERCP endoscopic retrograde cholangiopancreatography; EUS endoscopic ultrasound; GGT gamma-glutamyl transferase; RBO recurrent biliary obstruction; US ultrasound; MRI magnetic resonance imaging.

a Stent occlusion defined as elevation of inflammatory parameters/cholestasis evidence along with biliary dilation on imaging studies or endoscopic findings. Tokyo criteria [20].

b Stent migration defined as presence of completely/partially migrated stent at the time of endoscopic reintervention with evident stent dysfunction.

${ }^{c}$ Causes of stent occlusions: tumor ingrowth/overgrowth, biliary sludge, food impaction, hemobilia, kinking of CBD to stent). Tokyo criteria [20]. 
Time to RBO (t-BO) or stent patency is defined as the time from stent placement until the point when symptoms associated with occlusion or migration are observed.

$\mathrm{BRI}$ is defined as the need to perform a new therapeutic maneuver on the bile duct due to RBO. A distinction must be made between:

- Endoscopic biliary reintervention (e-BRI): endoscopic procedure with the aim of optimizing the transmural BD. It includes stent cleaning, stent change, additional stent insertion, or any other stentrelated endoscopic maneuver. For specific information, see Rescue options below.

- Radiological biliary reintervention (r-BRI): interventional radiological procedure with percutaneous access, with the aim of repermeabilizing the obstructed endoscopic BD.

\section{Safety reporting (AEs)}

$A E$ is any unwanted medical event, injury or clinical (e.g., signs, symptoms, or abnormal laboratory results) suffered by patients during the study, whether related to the endoscopic procedure or stent. All AEs (reported by medical staff or patients) must be documented.

All serious adverse events (SAEs) must be notified to the principal investigator within 3 days. In the event of a death, this notification will be made within less than 24 hours.

AEs will be rated as mild (hospitalization for 1-3 days), moderate (hospitalization for 4-10 days, ICU admission for 1 night, endoscopic/radiological intervention), severe (hospitalization >10 days, ICU admission $>1$ night, or necessity for surgery), or fatal, in accordance with the nomenclature for AEs in endoscopy (American Society for Gastrointestinal Endoscopy (ASGE) Workshops 2010) [21].

AEs associated with endoscopic biliary stents (e.g., pancreatitis, non-occlusion cholangitis, cholecystitis, bleeding, perforation, bile leakage) are defined by the standardized reporting system for endoscopic biliary stent placement (Tokyo criteria, 2018) $[20,21]$. It is important to note that RBO is not classified as an $\mathrm{AE}$, but as consequence of stent occlusion or migration.

Non-occlusion cholangitis will be defined as fever which continues longer than $24 \mathrm{~h}$ without dilation of the drained duct. It may require medication, hospitalization, or intervention.

The determination as to what is an AE related to the procedure or the medical device (procedure-related and stent-related) and what is not will be made by the medical team and local investigator, with final approval of the principal investigator (JG).

The AE will be chronologically differentiated as pre-procedure, intra-procedure, post-procedure, or early $A E$ (up to 14 days from the index procedure), and, finally, late AE (after 14 days) according the ASGE lexicon guideline [21]. Additionally, another phone call has been added at 30 days, to ensure that all stent-related AEs will be detected, as suggested by Tokyo criteria [20]. 
To clarify the causal relationship and grading of AEs, MEDDEV guidelines will be consulted. Additional file 2 includes all AE definitions based on the MEDDEV 2.12 guidelines (rev 8, July 2019) "Guidelines on medical devices: Clinical investigations: Serious Adverse event reporting under Directives 93/42 / EEC and 90/385 / EEC", regarding safety in clinical research with medical devices, which are determined according to the causal relationship and/or severity of AEs.

\section{Outcomes}

The primary outcome is the rate of RBO after index procedure. As noted above (Definition section), this is a direct indicator of stent dysfunction, and it is defined as a composite point of either biliary occlusion or stent migration.

The secondary outcomes include technical and clinical success, safety data and other patient-relevant outcomes (time to RBO, number of reinterventions, procedural time, and mortality rates).

Completed outcomes definitions are presented in Table 2.

\section{Sample size calculation}

The sample size calculation is based on the primary hypothesis of detecting significant differences in the rates of RBO and BRI between the LAMS and LAMS plus DPS groups (assessing for superiority).

- Null hypothesis $(\mathrm{H0}): \pi L A M S=\pi L A M S-D P S$

- Alternative hypothesis (H1): $\pi L A M S \neq \pi L A M S-D P S$

Based on recent published data, we estimate a recurrent biliary obstruction rate of $20 \%$ vs. $1 \%$ for the LAMS alone vs. LAMS-DPS groups, respectively up during follow-up period. To achieve a statistical power of $80 \%$ with a two-sided type I error of $5 \%$, a total of 80 patients ( 40 in each arm) is required to reject the null hypothesis. https://www.stat.ubc.ca/ rollin/stats/ssize/b2.html

Considering a $5 \%$ dropout rate, a final sample of 84 patients (42 patients in each arm) is needed.

\section{Statistical analysis}

Statistical analysis will be carried out with the R statistical software (version 3.6.2) supervised by the Biostatistics team of our research institute (IDIBELL).

Intention-to-treat and per-protocol analysis will be carried out. All study variables will be presented for stent groups and in total, using descriptive statistics in accordance with the nature of the variable. Thus, continuous variables will be described indicating the number of non-missing observations, mean, standard deviation, minimum, first quartile, median, third quartile, and maximum. The categorical variables will be described indicating the number of non-missing observations and the percentages of the different categories. 
Realization of the aim of the study will be analyzed by comparing the cumulative incidence of RBO (as a stent dysfunction parameter) in the LAMS cohort versus LAMS-DPS cohort using a Chi-Square test. The magnitude of the effect will be estimated through an incidence ratio and relative risk with $95 \%$ confidence interval. The main analysis will be replicated in an adjusted way using a binomial regression model. Age, sex, oncological status, and comorbidities will be taken as adjustment variables.

Safety will also be evaluated by describing the number and type of $A E$ in each study group, and the incidence will be compared using a Chi-square test. In addition, the survival of patients will be analyzed according to the two proposed strategies until the end of follow-up, describing the Kaplan-Meyer curves and comparing them using the LogRank test. The level of statistical significance is established at $p$-value $<0.05$.

\section{Subgroup analysis}

The main analysis will also be carried out in the following subgroups:

- Age $<65$ vs. Age $>65$.

- Presence of liver metastasis vs. Absence of liver metastasis.

\section{Data management}

Throughout the study the promoters will monitor the quality of the trial with special attention to protocol deviations and the quality of the data entered in the database. Scheduled video call will be performed by the primary investigator (JG) and/or research fellow (AGS). These audits will occur at 6 months postenrollment and at the end of the study period. At the end of the trial, a meeting will be held to consider the data management report. This report will describe the deviations from the protocol identified in each of the patients. These deviations will be classified as major or minor, and those patients with major deviations will be excluded from the protocol analysis. After the meeting, the suitability of the database for analysis will be considered, and the database will then be closed.

\section{Statistical analysis plan}

The statistical analysis plan will be finalized before the close of the database. This plan will include all the analysis described and others, mainly on the sensitivity of the results and the management of the missing data. If there is some deviation in the plan of statistical analysis regarding the main variable, an addendum to the protocol will be made. No changes will be made to the original analysis plan once the database is closed.

No interim analysis is planned given the short accrual time, relatively small sample size, and short followup period.

\section{Other considerations}

Rescue by e-BRI and cross-over: 
Depending on the initial endoscopic treatment carried out, in case of stent dysfunction or RBO, cross-over rescue treatment may be considered when the initial per protocol strategy fails:

- In LAMS cohort: adding a co-axial DPS through LAMS.

- In LAMS-DPS cohort: removing DPS and leaving LAMS or adding another coaxial stent, either fullycovered self-expandable metal stent or another DPS.

These potential cross-over options are considered in the e-BRI, described in a previous section (see definitions). In all these situations, the follow-up will be maintained until the end of the study in accordance with the protocol. Alternatively, if an endoscopic technique is not possible, other options (such as radiological intervention) will be offered.

Withdrawal

Withdrawal from the study will result from any AE or other clinical condition of the patient which, at the clinician's discretion, warrants such, or from pregnancy, or at the expressed wishes of the patient. Withdrawal from treatment will not mean suspension of the study, given that follow-up will be maintained until the end of the study in accordance with the protocol.

\section{Ethical aspects and confidentiality}

The protocol will be approved by the CEIC of each participating hospital as well as that of the coordinating center (Hospital Universitari de Bellvitge). The study researchers will carry out their tasks in compliance with ethical principles of clinical research established in the Declaration of Helsinki, and with the norms of Good Clinical Practices.

The study is considered a clinical trial with a low intervention level, since it compares two standard treatments with each other. Furthermore, all the medical devices used have CE marking with authorization for their use in endoscopic biliary drainage. For these reasons, the contracting of a specific civil liability policy is not required in accordance with the provisions of current legal regulations, complying with the assumptions established for coverage by the assistance policy.

Before inclusion of the patient in the trial, written informed consent will be requested. In relation to the study data, we will follow the provisions of Organic Law 3/2018 of December 5th on "Protection of Personal Data".

\section{Publication of results}

There is a commitment to publish the results of this study in high impact international journals, should the results be of sufficient scientific interest. No patient names will appear in any article, and no one, with the exception of the researchers in this study and the members of the hospital ethical committees, will have access to the data, in accordance with the Law on the Protection of Data of a Personal Nature.

\section{Discussion}


Currently, in vogue in the recent literature is reporting that EUS-BD (CDS type) using EE-LAMS is an effective and safe technique for biliary decompression in patients who failed $\operatorname{ERCP}[5,6,10-12,23]$. Some prospective studies or protocol trials have even been designed to assess CDS using LAMS as primary treatment modality for distal MBO over $\operatorname{ERCP}[2,7-9]$.

In fact, if it is shown that EUS-CDS is superior to ERCP, in terms of stent patency and safety, for the firstline drainage of $\mathrm{MBO}$, it is expected that the first-line BD method will be changed from ERCP to EUS-CDS, and that interruption of chemotherapy due to stent dysfunction could be avoided [9]. Additionally, even though percutaneous transhepatic BD (PTBD) may be considered as another alternative option after failed ERCP, some recent reports have claimed that EUS-BD with LAMS is superior to PTBD in terms of clinical success, safety, cost, and overall survival [24].

The introduction of these specific biliary LAMS represents a great technical improvement in EUS-BD of distal MBO $[3,4]$. The cautery-assisted stent eases the transmural technique, avoiding complexity, and failure risk linked to the devices exchanging or ostomy dilation, even without fluoroscopy guidance.

Even so, in two recent papers, including a meta-analysis, showing that LAMS and SEMS are comparable in terms of efficacy, safety, and reintervention rates for EUS-CDS, the authors concluded that further investigation is required, above all in adverse events $[13,23]$.

Stent dysfunction of biliary LAMS in EUS-CDS is one of the main concerns, and it has been reported in up to $26.3 \%$ of cases $[15,17,18]$. Unlike in pancreatic fluid collection [26], the prophylactic placement of a DPS within a biliary LAMS could offer two hypothetical advantages to prevent biliary AEs as RBO: (i) as an axis-orienting stent to maintain a non-perpendicular axis of the LAMS in the CBD; (ii) decreasing the risk of migration, limiting the possibility of LAMS slipping out. In a U.S. multicentre retrospective study, EI Chafic et al. proposed this technical variant, because RBO developed in significantly more patients with LAMS alone compared to LAMS plus DPS ( $50 \%$ vs. $11.8 \%$; $p=0.02)$ [23]. Similarly, two recent papers, including a large cohort from the UK and Ireland, revealed lower rates of cholangitis and BRI ( $\%$ vs $12.2 \%, p .03)$ in the DPS-LAMS group $[22,27]$. None of these previous studies were specifically designed for comparing the usefulness of the coaxial stent.

Recently, our group published a retrospective comparative study assessing the hypothetical benefit of a DPS within a biliary EE-LAMS in EUS-CDS. The DPS plus LAMS group had a higher clinical success rate, non-significantly lower AE rate, and longer significant procedural time. Curiously, the RBO rate was a little bit higher in the DPS-LAMS group [19]. Therefore, these results did not support this technical variant being used routinely, and it was a time-consuming approach.

Why the need to carry out a larger prospective and randomized study?

In case of confirmation that inserting a DPS within a LAMS during a EUS-CDS reduces the need for reinterventions, improves the biliary stent patency and prevents delays in the oncological management, this 
would surely have an impact on the oncological outcomes and survival rates. To date there is no prospective data about this issue.

The BAMPI trial will include seven referral centers, experts in EUS-guided transmural drainage, with proven experience in EUS-BD using EE-LAMS. Hospital Universitari de Bellvitge has leadership role in centralizing decisions in case of controversies, and in limiting heterogeneity.

In conclusion, this is the first multicentre randomized controlled trial designed to clarify whether the DPS plus LAMS strategy should be recommended routinely during a EUS-CDS in distal MBO. The results of this trial will have an impact on the clinical practice in treating patients with biliopancreatic malignancies.

\section{TRIAL STATUS}

Protocol of submitted version, number and date: Number 1.3; date December, 2021.

Recruitment: Start date November 17, 2020 and recruitment will be completed by October, 2022.

Revision chronology:

a- BAMPI, August 2020, original: version 1.0, first draft of the study protocol.

b- BAMPI, November 2020, amendment $n^{\circ} 1$ : version 1.1.

- Main amendments: (i) to clarify: The study is considered a clinical trial with a low intervention level. To contract a specific civil liability policy is not required in accordance with the provisions of current legal regulations.

c- BAMPI November, 2021, amendment n²: version 1.2.

- Minor changes: addition of a new rescue variant technique. If CDS is not possible (due to interposing vessels, significant distance), gallbladder drainage will be considered.

d-BAMPI December 2021, amendment n³: version 1.3 - definitive.

- Minor changes: extension of the recruitment period (until October 2022).

\section{List Of Abbreviations}

AE: Adverse event

ASA: American Society of Anesthesiologists' classification

BD: Biliary drainage

BRI: Biliary reintervention 
CBD: Common bile duct

CEIC: Clinical research ethics committees

CRF: Case report form

DPS: Double-pigtail plastic stent

EE: Electrocautery-enhanced

EUS: Endoscopic ultrasound

EUS-BD: Endoscopic ultrasound-guided biliary drainage

IDIBELL: Bellvitge Biomedical Research Institute

LAMS: Lumen-apposing metal stent

MRI: Magnetic resonance imaging.

MBO: Malignant biliary obstruction

PTBD: percutaneous biliary drainage

RBO: Recurrent biliary obstruction

SEED: Spanish Society of Digestive Endoscopy

TA: Tissue acquisition

\section{Declarations}

- Ethics approval and consent to participate: Ethical approval was obtained from the Comité Ético de Investigación Clínica del Hospital Universitari de Bellvitge -IDIBELL (Barcelona; ref approval no. ICPS024/20), on November 5, 2020. Written and oral informed consent will be obtained from all participants before enrolment in the study. Data collected during the course of the research will be kept strictly confidential and only accessed by investigators. An individual trial identification number and participant details will be stored on a secure database. Any subsequent amendments of the protocol need to be approved by the relevant ethical bodies before implementation. Participants will also be asked for permission for the research team to share relevant data with investigators from universities taking part in the research or from regulatory authorities, where relevant.

- Consent for publication: not applicable.

- Availability of data and material: minimal dataset necessary to interpret the findings available from the corresponding author on reasonable request. 
- Competing interests:

- A Garcia-Sumalla is a PhD student at the Faculty of Medicine and Health Sciences, University of Barcelona, and this paper is part of his doctoral project.

- JB Gornals acts as a PhD Director; is a consultant for Boston Scientific and has received a research grant from this company.

- M Pérez-Miranda is consultant and speaker for Medtronic, Olympus, Taewoong, and M.I.Tech

- E Vazquez-Sequeiros is a consultant for Boston Scientific

- JR Aparició is a consultant for Boston Scientific.

- The other authors declare that they have no competing interests.

\section{- Funding:}

This study received a grant (Fundacion SEED) from the Sociedad Española de Endoscopia Digestiva in 2020, and a grant (beca d'iniciació) from the Societat Catalana de Digestologia in 2021. The funding bodies have no role in the trial design or interpretation of the data.

\section{- Authors' contributions:}

AGS, CT, and JBG drafted the manuscript. AGS, CL, VS, RPS, EVS, JRA, CSH, DLG, XA, MC, TB, JRF, BM, $\mathrm{CC}, \mathrm{SS}, \mathrm{BL}, \mathrm{MPM}$, and JBG provided a critical review, and, as investigators at each institution, will promote enrolment of patients. JBG and AGS registered the study. AGS, JBG, and CT contributed to the statistical analysis. SV offered scientific advice on the publication of a protocol. AGS, JBG, MPM, and CLA participated in the design of the study. JBG conceived the project. AGS and JBG designed the study, revised the manuscript, and approved the final submission. All authors read and approved the final manuscript.

\section{- Acknowledgements:}

The Asociación Española de Gastroenterología (AEG) for providing us with REDCap platform. This platform is free of charge, and is a secure web-based application designed to support data capture for research studies.

Enric Sospedra, secretary of the Comité Ètic i d'Investigació Clínica (CEIC) de l'Hospital Universitari de Bellvitge, for his generous advice on drafting the responses to co-centers CElm amendments.

The institutions and scientific societies that supported this project with grants (Sociedad Española de Endoscopia Digestiva and Societat Catalana de Digestologia).

Sandra Maisterra, Julio G Velasquez-Rodriguez, and Claudia F Consiglieri of Hospital 
Universitari de Bellvitge, who will assist in the enrolment of patients.

\section{Steering Committee:}

Manuel Perez-Miranda, Hospital Universitario Rio Ortega, Valladolid, Spain.

Carme Loras, Hospital Mutua Terrassa, Catalonia, Spain.

Joan B Gornals, Hospital Universitari de Bellvitge, Barcelona, Catalonia, Spain.

\section{References}

1. Puga $M$, Pallarès N, Velásquez-Rodríguez J, García-Sumalla A, Consiglieri CF, Busquets J, Laquente B, Calvo M, Fabregat J, Castellote J, Gornals JB. Endoscopic biliary drainage in unresectable biliary obstruction: the role of endoscopic ultrasound-guidance in a cohort study. Rev Esp Enferm Dig 2019; 111: 683-689.

2. Chen YI, Callichurn K, Chatterjee A, Desilets E, Fergal D, Forbes N, Gan I, Kenshil S, Khashab MA, Kunda R, Lam E, May G, Mohamed R, Mosko J, Paquin SC, Sahai A, Sandha G, Teshima C, Barkun A, Barkun J, Bessissow A, Candido K, Martel M, Miller C, Waschke K, Zogopoulos G, Wong C; ELEMENT trial and for the Canadian Endoscopic Research Collaborative (CERC). ELEMENT TRIAL: study protocol for a randomized controlled trial on endoscopic ultrasound-guided biliary drainage of first intent with a lumen-apposing metal stent vs. endoscopic retrograde cholangio-pancreatography in the management of malignant distal biliary obstruction. Trials. 2019; 20: 696.

3. Itoi T, Binmoeller KF. EUS-guided choledochoduodenostomy by using a biflanged lumen-apposing metal stent. Gastrointest Endosc. 2014; 79: 715.

4. Gornals JB, Consiglieri C, Gallarreta V, Busquets J, Catala I, Laquente B. Single-session fluoroless endoscopic ultrasound-guided fine-needle aspiration and choledochoduodenostomy with a biliary lumen-apposing stent. Endoscopy. 2015;47:418-419.

5. Anderloni A, Anderloni A, Fugazza A, Troncone E, Auriemma F, Carrara S, Semeraro R, Maselli R, Di Leo M, D'Amico F, Sethi A, Repici A. Single-stage EUS-guided choledochoduodenostomy using a lumen-apposing metal stent for malignant distal biliary obstruction. Gastrointest Endosc. 2019; 89: 69-76.

6. Jacques J, Privat J, Pinard F, Fumex F, Valats JC, Chaoui A, Cholet F, Godard B, Grandval P, Legros R, Kerever S, Napoleon B. Endoscopic ultrasound-guided choledochoduodenostomy with electrocauteryenhanced lumen-apposing stents: A retrospective analysis. Endoscopy. 2019; 51: 540-547.

7. Paik WH, Lee TH, Park DH, Choi JH, Kim SO, Jang S, Kim DU, Shim JH, Song TJ, Lee SS, Seo DW, Lee SK, Kim MH. EUS-guided biliary drainage versus ERCP for the primary palliation of malignant biliary obstruction: a multicenter randomized clinical trial. American Journal of Gastroenterology. 2018; 113: $987-97$. 
8. Park JK, Woo YS, Noh DH, Yang J il, Bae SY, Yun HS, Lee JK, Lee KT, Lee KH. Efficacy of EUS-guided and ERCP-guided biliary drainage for malignant biliary obstruction: prospective randomized controlled study. Gastrointestinal Endoscopy. 2018; 88: 277-82.

9. Itonaga M, Kitano M, Yoshikawa T, Ashida R, Yamashita Y, Hatamaru K, Takenaka M, Yamazaki T, Ogura T, Nishioka N, Sakai A, Masuda A, Shiomi H, Shimokawa T. Comparison of endoscopic ultrasound-guided choledochoduodenostomy and endoscopic retrograde cholangiopancreatography in first-line biliary drainage for malignant distal bile duct obstruction: A multicenter randomized controlled trial. Medicine (Baltimore). 2021 Mar 26;100(12):e25268.

10. Krishnamoorthi R, Dasari CS, Thoguluva V, Priyan H, Jayaraj M, Law J, Larsen M, Kozarek R, Ross A, Irani S. Effectiveness and safety of EUS-guided choledochoduodenostomy using lumen-apposing metal stents ( LAMS): a systematic review and meta-analysis. Surg Endosc 2020; 34: 2866-2877.

11. Jain D, Shah M, Patel U, Sharma A, Singhal S. Endoscopic ultrasound guided choledochoenterostomy by using lumen apposing metal stent in patients with failed endoscopic retrograde cholangiopancreatography: a literature review. Digestion. 2018; 98:1-10.

12. Mohan BP, Shakhatreh M, Garg R, Ponnada S, Navaneethan U, Adler DG. Efficacy and safety of endoscopic ultrasound-guided choledochoduodenostomy: a systematic review and metanalysis. Journal of Clinical Gastroenterology. 2019; 53: 243-50.

13. Amato A, Sinagra E, Celsa C, Enea M, Buda A, Vieceli F, Scaramella L, Belletrutti P, Fugazza A, Cammà C, Radaelli F, Repici A, Anderloni A. Efficacy of lumen-apposing metal stents or self-expandable metal stents for endoscopic ultrasound-guided choledochoduodenostomy: a systematic review and metaanalysis. Endoscopy. 2021; 53: 1037-1047.

14. Han SY, Kim SO, So H, Shin E, Kim DU, Park DH. EUS-guided biliary drainage versus ERCP for first-line palliation of malignant distal biliary obstruction: A systematic review and meta-analysis. Scientific Reports. 2019 Dec 1;9(1).

15. Tsuchiya T, Teoh AYB, Itoi T, Yamao K, Hara K, Nakai Y, Isayama H, Kitano M. Long-term outcomes of EUS-guided choledochoduodenostomy using a lumen-apposing metal stent for malignant distal biliary obstruction: a prospective multicenter study. Gastrointestinal Endoscopy [Internet]. 2018; 87: 1138-1146.

16. Garcia-Sumalla A, Bazaga S, Gornals JB. EUS-guided biliary rendezvous as an emergent rescue after failed choledochoduodenostomy using a lumen-apposing metal stent. VideoGIE. 2021 Jun 1; 6: 263-5.

17. Mosquera-Klinger G, de la Srna C, Perez-Miranda M. Endoscopic sump syndrome secondary to EUSguided choledochoduodenostomy with a lumen-apposing metal stent. Rev Esp Enferm 111:74-76.

18. Gornals JB, Consiglieri CF, Bergamino MA. Double pigtail for preventing ascending cholangitis after endoscopic ultrasonography-guided choledochoduodenostomy with lumen-apposing metal stent. Dig Endosc. 2016; 28: 100.

19. Garcia-Sumalla A, Loras C, Guarner-Argente C, Velasquez-Rodriguez JG, Andujar X, Salord S, Busquets J, Tebe C, Laquente B, Gornals JB. Is a coaxial plastic stent within a lumen-apposing metal 
stent useful for the management of distal malignant biliary obstruction? Surg Endosc. 2021; 35 : 4873-4881.

20. Hamada T, Nakai Y, Isayama H. TOKYO criteria: Standardized reporting system for endoscopic biliary stent placement. Gastrointestinal Interv. 2018; 7: 46-51. DOI:10.18528/gii180016.

21. Cotton PB, Eisen GM, Aabakken L, Baron TH, Hutter MM, Jacobson BC, Mergener K, Nemcek A Jr, Petersen BT, Petrini JL, Pike IM, Rabeneck L, Romagnuolo J, Vargo JJ. A lexicon for endoscopic adverse events: report of an ASGE workshop. Gastrointestinal Endoscopy. $2010 ; 71: 446-454$.

22. On W, Paranandi B, Smith AM, Venkatachalapathy S v., James MW, Aithal GP, Varbobitis I, Cheriyan D, McDonald C, Leeds JS, Nayar MK, Oppong KW, Geraghty J, Devlin J, Ahmed W, Scott R, Wong T, Huggett MT. EUS-guided choledochoduodenostomy with electrocautery-enhanced lumen-apposing metal stents in patients with malignant distal biliary obstruction: multicenter collaboration from the United Kingdom and Ireland. Gastrointestinal Endoscopy. 2021 Oct 9; S0016-5107(21)01702-8. doi: 10.1016/j.gie.2021.09.040.

23. el Chafic AH, Shah JN, Hamerski C, Binmoeller KF, Irani S, James TW, Baron TH, Nieto J, Romero RV, Evans JA, Kahaleh M. EUS-guided choledochoduodenostomy for distal malignant biliary obstruction using electrocautery-enhanced lumen-apposing metal stents: First US, multicenter experience. Dig Dis Sci. 2019; 64: 3321-3327.

24. Ginestet C, Sanglier F, Hummel V, Rouchaud A, Legros R, Lepetit H, Dahan M, Carrier P, Loustaud-Ratti V, Sautereau D, Albouys J, Jacques J, Geyl S. EUS-guided biliary drainage with electrocauteryenhanced lumen-apposing metal stent placement should replace PTBD after ERCP failure in patients with distal tumoral biliary obstruction: a large real-life study. Surg Endosc. 2021 Oct 4. doi: 10.1007/s00464-021-08653-1.

25. de Benito Sanz M, Nájera-Muñoz R, de la Serna-Higuera C, Fuentes-Valenzuela E, Fanjul I, Chavarría C, García-Alonso FJ, Sanchez-Ocana R, Carbajo AY, Bazaga S, Perez-Miranda M. Lumen apposing metal stents versus tubular self-expandable metal stents for endoscopic ultrasound-guided choledochoduodenostomy in malignant biliary obstruction. Surg Endosc. 2021; 35: 6754-6762.

26. Puga M, Consiglieri CF, Busquets J, Pallarès N, Secanella L, Peláez N, Fabregat J, Castellote J, Gornals JB. Safety of lumen-apposing stent with or without coaxial plastic stent for endoscopic ultrasound-guided drainage of pancreatic fluid collections: a retrospective study. Endoscopy. 2018; 50: 1022-1026.

27. Chin JY-L, Seleq S, Weilert F. Safety and outcomes of endoscopic ultrasound-guided drainage for malignant biliary obstruction using cautery-enabled lumen-apposing metal stent. Endoscopy International Open. 2020;08: E1633-8.

\section{Figures}




\begin{tabular}{|c|c|c|c|c|c|c|c|c|c|c|}
\hline & & \multicolumn{9}{|c|}{ STUDY PERIOD } \\
\hline & Enrolment & Allocation & & & & & & & & Close-out \\
\hline TIMEPOINT & $-t_{1}$ & 0 & $\mathrm{~T}_{1}$ & $24 \mathrm{~h}$ & $14 d \pm 2 d$ & $30 d \pm 2 d^{a}$ & $90 d \pm 5 d$ & $180 d \pm 5 d$ & $270 d \pm 5 d$ & 1-year \\
\hline \multicolumn{11}{|l|}{ ENROLMENT: } \\
\hline Informed consent & $\mathrm{x}$ & & & & & & & & & \\
\hline $\begin{array}{l}\text { Clinical history, } \\
\text { baseline blood test }\end{array}$ & $\mathrm{x}$ & & & & & & & & & \\
\hline $\begin{array}{l}\text { Eligibility screen } \\
\text { (> or }<65 \text { y, liver } \\
\text { metastases) }\end{array}$ & $x$ & & & & & & & & & \\
\hline Allocation & $x$ & $\mathrm{x}$ & & & & & & & & \\
\hline \multicolumn{11}{|l|}{$\begin{array}{l}\text { INTERVENTION } \\
\text { (EUS-BD): }\end{array}$} \\
\hline LAMS alone & & & $\mathrm{x}$ & & & & & & & \\
\hline LAM plus DPS & & & $\mathrm{x}$ & & & & & & & \\
\hline \multicolumn{11}{|l|}{ ASSESSMENTS: } \\
\hline Visit & $\mathrm{X}$ & & & $\mathrm{X}$ & & & & & & \\
\hline Telephone contact & & & & & $\mathrm{X}$ & $\mathrm{X}$ & $\mathrm{X}$ & $\mathrm{X}$ & $\mathrm{X}$ & $\mathrm{X}$ \\
\hline INR/platelets & $x$ & & & & & & & & & \\
\hline Blood test & $x$ & & & $\mathrm{x}$ & $x$ & $X^{3}$ & $\mathrm{X}$ & $\mathrm{X}$ & $x$ & $x$ \\
\hline Symptomatology & $\mathrm{x}$ & & & $\mathrm{x}$ & $\mathrm{x}$ & $\mathrm{x}$ & $\mathrm{x}$ & $\mathrm{x}$ & $\mathrm{x}$ & $\mathrm{x}$ \\
\hline $\begin{array}{l}\text { Primary outcome } \\
\text { (RBO) }\end{array}$ & & & & & $x$ & $x$ & $x$ & $x$ & $x$ & $x$ \\
\hline $\begin{array}{l}\text { Secondary } \\
\text { Outcomes: }\end{array}$ & & & $\mathrm{x}$ & $x$ & $x$ & $x$ & $x$ & $x$ & $x$ & $x$ \\
\hline -Technical Success & & & $x$ & & & & & & & \\
\hline -Clinical Success & & & & & $x$ & & & & & \\
\hline -Adverse events & & & $\mathrm{x}$ & $\mathrm{x}$ & $x$ & $\mathrm{x}$ & $x$ & $x$ & $\mathrm{x}$ & $\mathrm{x}$ \\
\hline -BRI & & & & $\mathrm{x}$ & $\mathrm{x}$ & $\mathrm{x}$ & $\mathrm{x}$ & $x$ & $\mathrm{x}$ & $\mathrm{x}$ \\
\hline Medication & $\mathrm{x}$ & & & $\mathrm{x}$ & $x$ & $\mathrm{x}$ & $x$ & $x$ & $\mathrm{x}$ & $\mathrm{x}$ \\
\hline
\end{tabular}

\section{Figure 1}

Enrollment, interventions, and assessment in the BAMPI trial (SPIRIT-Figure).

a Mandatory only in case of NOT meeting clinical success criteria on previous visit (14 day).BRI biliary reintervention; $d$ days; EUS-BD, endoscopic ultrasound-guided biliary drainage. 


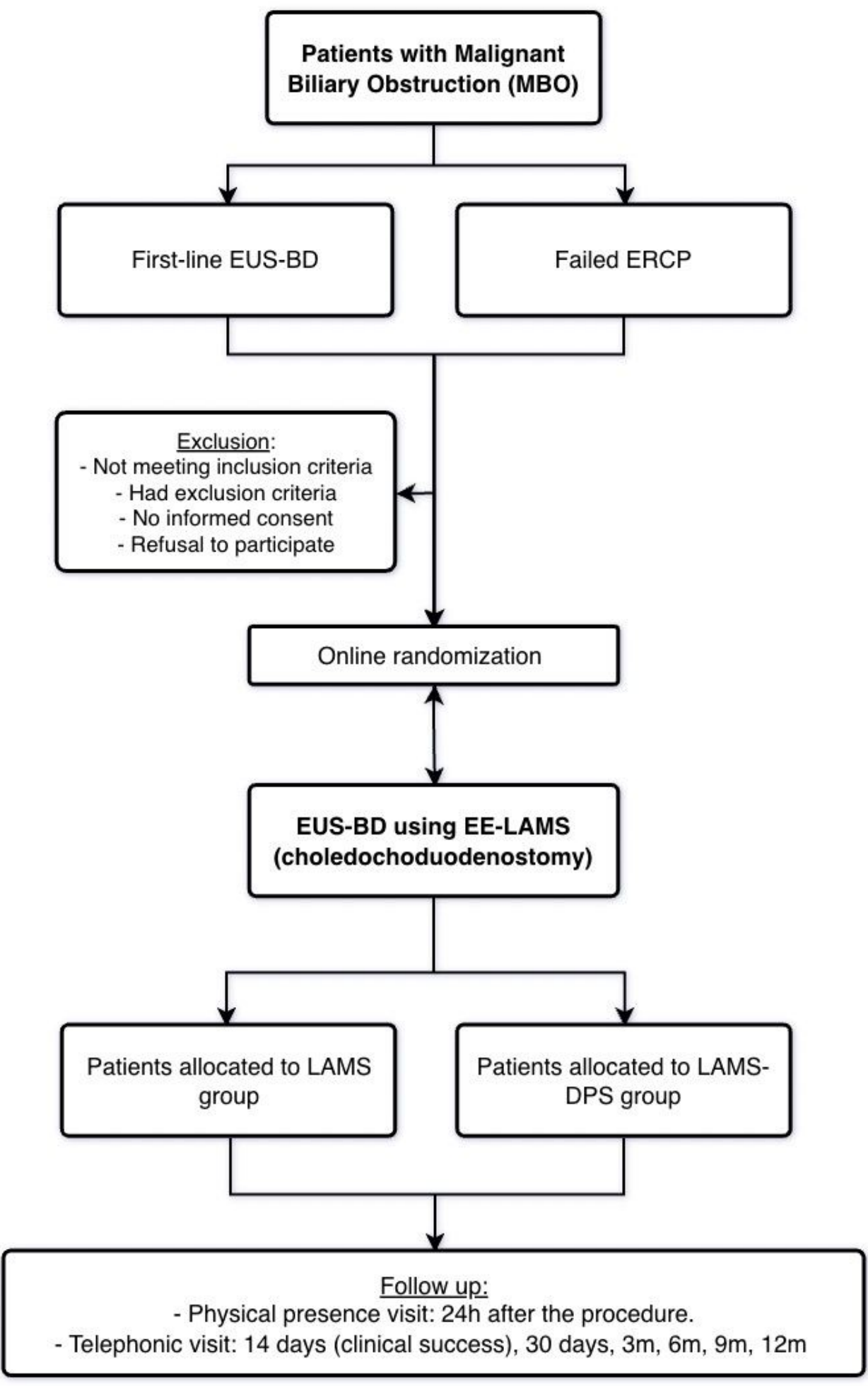

Figure 2

BAMPI trial Flowchart.

DPS double-pigtail plastic stent, ERCP endoscopic retrograde cholangio-pancreatography, EUS-BD endoscopic ultrasound guided biliary drainage, EE-LAMS, electrocautery-enhanced, lumen-apposing metal stent. 


\section{Supplementary Files}

This is a list of supplementary files associated with this preprint. Click to download.

- appendix1SPIRITchecklist.BAMPIdef.doc

- appendix2BAMPISafetydefinitionsAE.docx 